\title{
Seasonal Variation in Gut Microbiota Composition: Comparative Analysis of Siberian Musk Deer (Moschus moschiferus) and Forest Musk Deer (Moschus berezovskii)
}

\section{Rina Su}

Inner Mongolia Agricultural University

Myagmarsuren Erdenedalai

Inner Mongolia Agricultural University

Menggen Dalai

Affiliated Hospital of Inner Mongolia Medical College

\section{Batkhuu}

Institude of traditional medicine and technology

Chimedragchaa Chi

institude of traditional medicine and technology

Surong Hasi ( $\nabla$ surong@imau.edu.cn )

Inner Mongolia Agricultural University

Research article

Keywords: gut microbiota, seasonal variation, 16s rRNA, Siberian musk deer, Forest musk deer

Posted Date: June 5th, 2020

DOI: https://doi.org/10.21203/rs.3.rs-33043/v1

License: (c) (i) This work is licensed under a Creative Commons Attribution 4.0 International License.

Read Full License 


\section{Abstract}

Background: Musk deer is famous for its secretion of musk, but due to various factors that the musk deer population has dropped sharply and it is difficult to expand herd sizes. In light of the rapid development of agriculture and technology, as well as the urgent need for wildlife conservation, research on the gut microbiota of musk deer is warranted. The gut microbiota impacts host health is shaped by dietary, environmental, and host factors.

Results: We studied the gut microbiota in Siberian musk and Forest musk deer using high-throughput sequencing of the V3-V4 region of the 16S rRNA gene. Fecal samples were collected during two seasons to determine associations between community diversity, composition, and seasonal factors. The results show that the Firmicutes and Bacteroidetes were the predominant phyla in both species, their relative abundance was significantly different with species and seasonal factors. The Siberian musk deer population sampled in this study had a wider feeding range and no dietary limitations. Affected by the richness and type of food sources, the microbial a-diversity is higher in summer than winter. In the siberian musk deer have only Tenericutes significant difference with the season change.

Conclusions:The gut bacterial composition of these two musk deer is significantly different, and the abundance of core bacteria is affected by seasonal factors. This study is the first to analyze the composition of the gut microflora in Siberian musk deer, and these results provide a foundation for improving feeding for musk deer populations and further studies investigating the environmental impact of musk deer.

\section{Background}

Both Siberian musk deer (Moschus moschiferus; SMD) and forest musk deer (Moschus berezovskii; FMD) belong to the family Moschidae and genus Moschus. They are generally solitary, as well as have a stocky body with short, thin front legs and longer, more powerful hind legs. Siberian musk deer is a medium-sized musk deer ( $80 \sim 95 \mathrm{~cm}$ head-body length and $8 \sim 13 \mathrm{~kg}$ in weight), while forest musk deer is one of the smaller musk deer (70 cm head-body length and $7 \sim 9 \mathrm{~kg}$ in weight). Male musk deer is different from the true deer of the Cervidae family because they do not have antlers. On the contrary, they have two tusk-like canine teeth that stick out below the lower jaw. The Siberian musk deer are native to Russia, Kazakhstan, China, Mongolia, and the Koreas [1]. Their body fur is long, dense and dark brown, and mainly grey on the head. Meanwhile, the Siberian musk deer have some brown areas at the crown and around the long ears that are the same as hare[1-4]. More than 5,000 years of history, the musk has been widely used in the production of Chinese traditional medicine and perfume. In the past, most of the musk were obtained by killing active animals to acquire the entire sac [2, 3]. So Moschusgenue spp. populations are declining due to hunting. The species are listed under CITES Appendix II, and is protected (Very Rare) under part 7.1 of the Law of the Mongolian Animal Kingdom (2000). It is also on the 2014 Chinese Red List as Critically Endangered A1acd+B1ab (i, ii, iii), and is contained on the China Key List-II $[5,6]$. 
Gut microbial composition and function in wild animals has been widely studied in recent years [7, 8]. A number of vital host functions depend on the gut microbiota, which is involved in the synthesis of nutrients and systemic immune regulation [9]. The gut microbiota is a complex ecosystem that is regulated by many factors. The composition of gut microbiota is altered by the host, feed, lifestyle, antibiotics and seasonal changes [10-13]. Studies in horses[14], mice [15], pandas [16], humans [17], and forest musk deer [18] have shown seasonal shifts in gut microbiome structure. These alterations could be caused by the diet. In summer, the rainfalls increased, and the density of plants will be improved. It means that musk deer have sufficient food. However, the food supply will be decreased in winter because of severe weather. Some gut bacteria are opportunistic pathogens, and bacterial overgrowth or an altered community structure are associated with multiple diseases $[11,19]$. Siberian musk deer and Forest musk deer have a high rate of disease incidence, especially diseases caused by a bacterial infection, and the mortality rate of diseased animals is high. These infections can be mediated by environmental or hostderived bacteria $[20,21]$. The precise role of the gut microbiota on animal health and disease remains unclear and more foundational work is required. The Siberian musk and forest musk deer are herbivorous ruminants. Although the microbiota can be an important tool for assessing animal health, no reports to date have studied these factors in Siberian musk deer.

We characterized the seasonal variation in the gastrointestinal microbiota of Siberian musk deer and forest musk deer using high-throughput sequencing of the 16S rRNA gene in fecal samples. According to the research results of Hu et al [18] that the influence of seasonal factors on the gut microbiota of forest musk deer, we selected representative winter and summer seasons for this research. The main objectives of present study are: (1) to compare the composition and diversity gut microbiota of two species; (2) to determine seasonally (i.e., summer and winter) variations in the microbial community composition and disease of Siberian musk deer; (3) to examine the changes in signaling pathways and functional genes, and; (4) to discuss their potential functions in the diagnosing diseases of the digestive system and for improving feeding methods.

\section{Results}

\section{Quality filtering and validation of the dataset}

There was an average of 909,993 reads (average length $=428.38$ bps; Table S1) obtained from each sample after quality filtering. There were 1,627 OTUs detected, and each sample had between 410 and 846 OTUs (Table S2). Individually rarefaction curves showed that the sequencing depth was adequate for the study (Fig S1). Good's coverage showed that 28 samples had between 99.28 to $99.62 \%$ coverage (mean $=99.44 \%$; Table S2). The results showed that the number of OTUs increased rapidly until it gradually reached a plateau. It shows that the amount of sequencing data is large enough, and the increase of sequencing data can not find more OTU, which can reflect the information of most microbial species in the sample.

Core bacterial communities and composition difference in the two species 
There were 18 phyla, 25 classes, 59 orders, 109 families, and 269 genera identified in the two musk deer species. There were 658 OTUs that were present in all groups. There were 92, 56, 101, and 12 OTUs that appeared in each sample of the FS, FW, SS, and SW groups, respectively (Fig 1A; FMD in summer and winter: FS and FW; SMD in summer and winter: SS and SW ).

The classification of sequences from the samples resulted in six different phyla was identified, including Firmicutes, Bacteroidetes, Spirochaetes, Verrucomicrobia, Tenericutes, and Proteobacteria (Fig 1B). We considered these taxa that were shared by all individuals in a group to be the core bacterial community for that group. Firmicutes (FMD: $52 \pm 8.427$ to $64.03 \pm 11.69$, SMD: $84.57 \pm 6.305$ to $89.86 \pm 3.094$ ) and Bacteroidetes (FMD: $38.17 \pm 7.489$ to $29.09 \pm 8.978$, SMD:12.78 \pm 6.113 to $8.89 \pm 2.302$ ) were core and dominant community members for both musk deer species. The relative abundances of Firmicutes were significantly higher in SMD than in FMD, and this corresponded to decreases in the relative abundances of Bacteroidetes (Fig 1BषTable 2). Spirochaetes and Verrucomicrobia were only found in forest musk deer (Fig 1B). Also, the difference in gut microbiota composition between the two musk deer can be clearly observed according to the Heatmap. Our results indicated the existence of two core taxa at the class level (Clostidiales and Bacteroidales) (Fig S2). At the order level, core gut bacterial included Ruminococcaceae, Prevotellaceae, Bacteroidaceae, Lachnospiraceae, Rikenellaceae, and Christensenellaceae (Fig S3), in addition to this six core taxa at the family level(Fig S4). These results indicated that the composition of the microbial community may be related to different physiological characteristics in the gut of musk deer.

The alpha diversity of forest musk deer was higher than that of Siberian musk deer based on the Shannon and Chao indices (FMD: Shannon index $=4.662 \pm 0.42366$, Chao $=819.29 \pm 138.02, p=0.4306$; SMD: Shannon $=4.5207 \pm 0.55078$, Chao $=761.44 \pm 137.5, p=0.4014$; Table S3;Fig 2A-2B ), it is concluded that the community abundance and community diversity of the forest musk deer are higher than the siberian musk deer, but there is no significant difference. There were differences in the beta diversity of the SMD and FMD communities PCoA analysis (Fig 3). ANOSIM analysis reveals intergroup differences in bacterial communities between two musk deer species $(R=0.6643, p=0.001)$. The results of ANOSIM analysis and PCoA analysis mutually verified the differences of bacterial communities between the two musk deer species.

\section{Seasonal differences in bacterial communities}

The alpha diversity of the bacterial community in the summer was higher than in the winter for the two musk deer species(Shannon index; FS:4.8901 \pm 0.2506 , SS: $4.8339 \pm 0.50796, F W: 4.4339 \pm 0.4478$, SW: 4.2076 \pm 0.43399$)$. There was a significant difference in Shannon diversity index between the two seasons for FMD ( $p=0.02113, \mathrm{Q}=0.02536)$, but not for SMD ( $p=0.1939, \mathrm{Q}=0.2327 ;$ Fig $2 \mathrm{C}$; Table S3). The Chao index have significant different in SMD (Chao: $p=0.03038, Q=0.06077$; Fig 2D). At the phyla level, there were 8 taxa (Firmicutes, Bacteroidetes, Actinobacteria, Cyanobacteria, Patescibacteria, Liritimatiellaeota, Lentisphaerae, and Elusimicrobia )whose relative abundance was significantly different between seasons in FMD; at the same time, and only one taxon (Tenericutes) whose relative abundance was significantly 
different between seasons in SMD (Table 2; Fig 4A; Fig S5). It is worth mentioning that the Firmicutes/Bacteroidetes ratio changed with the season in two musk deer.

We are much interested in musk deer gut microbiota is to determine whether specific microbiota could be the change with different season factors. To identify specific microbial candidates present in two specise seasons, LEFse (LDA score $\geq 4$ ) analysis were used to determine specific biomarker belonged to different taxonomy (Fig 4B) from phylum to genus. The result revealed that twelve families (Bacteroidia, Bacteroidales, Bacteroidetes, Prevotellaceae, Prevotellaceae_UCG_004, Bacteroides, Bacteroidaceae, Verrucomicrobia, Verrucomicrobiae, Verrucomicrobiales, Akkermansia, and Akkermansiaceae) were significantly enriched in the FS group, one family (Coprococcus) was significantly enriched in the FW group, two families (Ruminococcaceae_UCG_010 and Ruminococcaceae_UCG_013) were significantly enriched in the SS group, and seven families (Firmicutes, Clostridia, Clostridiales, Lachnospiracea, Christensenellaceae_R_7_group, Christensenellaceae, and unclassified_Lachnospiraceae) were significantly enriched in the SW group (Fig 4B).

\section{Predicted functions of the microbial communities}

There were significant differences in the functional capacity of SMD and FMD microbial communities. Specifically, three KEGG pathways (Environmental Information Processing, Cellular Processes, and None) were enriched in SMD samples, and five KEGG pathways (Human Diseases, Organismal Systems, Metabolism, Genetic Information Processing, and Unclassified) were enriched in FMD samples at Level 1 (Fig 5A). Also, two groups of samples were significant differences in thirty-three KEGG pathways (Level 2; $p<0.005$ ). Among them, seven KEGG pathways (Transcription, Membrane Transport, Cell Motility, Signal Transduction, Cellular Processes and Signaling, Environmental Adaptation, and Carbohydrate Metabolism) were significantly enriched in SMD samples. Twenty-six KEGG pathways (Translation, Replication and Repair, Nucleotide Metabolism, "Folding, Sorting and Degradation", Enzyme Families, and Metabolic Diseases, et a) were significantly enriched in FMD samples (Fig 5B).At level 3, a functional comparison of 143 extremely significant differences $(p<0.001)$ was performed. Among them, the SMD group enriches thirty-five functions, and the FMD group enriches other functions. The results also reflect differences in transcription and metabolism between the two groups of animals (Fig S6).

There was a significant difference in predicted microbiota function in SMD between summer and winter. The relative abundance of genes (Level 1) involved in Metabolism, Organismal Systems, and Human Diseases was significantly different in two seasons (Fig 5C). The relative abundance of gene families(Level 2) involved in Xenobiotics Biodegradation and Metabolism, Endocrine System, and Metabolism of Cofactors and Vitamins was significantly higher in the SS samples than in the SW samples (Fig 5C). Besides, the Influenza A, Toxoplasmosis, Colorectal cancer, Viral myocarditis, and Smell cell lung cancer functions (Level 3) were significantly enriched in the SS group (Fig 5C).

\section{Discussion}


A high-fiber diet coupled with long-term evolutionary adaptation has resulted in a large number of symbiotic bacteria in the gastrointestinal tract of herbivores to help the body digest and absorb nutrients. At the same time, it also gives the ruminant gastrointestinal flora a distinct feature and a similar flora structure. Our data shows the difference in microbial communities between musk deer species as well as the seasonal variations in the microbial communities of each deer species. The a-diversity of the gut microbiota in FMD samples was significantly different in two seasons, and the diversity lower in winter than in summer (Shannon index: $p<0.05$; Chao index: $p<0.05$ ); meanwhile, the diversity of the gut microbiota in SMD samples was not significantly different between seasons. Gut microbial diversity in mice[22] and humans [23] is significantly lower in diseased subjects. It shows that the diversity of gut microbiota is related to animal health. Also, many studies have shown that modern lifestyles have a negative impact on gut microbiota diversity that the simplification of diet structure, the use of antibiotics and the reduction of outdoor activities are some of the factors that cause the diversity of gut microbiota to decrease $[17,24,25]$. In winter, survival strategies are most important for wild populations with low energy intake and high energy consumption. In terms of food types, there are fewer types of food to choose from in winter, and it is more difficult to eat the herbs on the surface, which results in a single diet for Siberian musk deer. It is speculated that the less variety of food is a factor that causes the lower gut microbiota diversity of Siberian musk deer.

The core bacterial phyla in both musk deer species were Firmicutes and Bacteroidetes, which is consistent with previous studies of the microbiota of musk deer $[18,26,27]$ and other ruminants [28- 30]. The phyla are found in the guts of many mammals, which suggest the importance of these bacteria in the gut ecosystem [31]. Firmicutes are predominant cellulolytic bacteria that able to degrade fiber and form short-chain fatty acids (SCFAs), which are utilized by the host [32-34]. Ruminants cannot produce the necessary cellulolytic enzymes to degrade fiber and must rely on bacteria to produce these enzymes [35]. Certain Bacteroidetes species are specialists that degrade high molecular weight organic material (i.e., carbohydrates and proteins), and the genomes of these species are known to contain large numbers of CAZymes [36]. The seasonal variations in microbiome composition in our study are likely due to seasonal changes in diets. Diet is an important factor that shapes gut microbial communities [37-39]. Specifically, the ratio of fiber to carbohydrates in diets can impact microbiome composition in ruminants [40]. For example, the content of animal protein and saturated fatty acids are related to the abundance of Actinomycetes, while the abundance of carbohydrates, monosaccharides (glucose and fructose) and the abundance of Pseudomonas and Prevotella are highly correlated. Bacteroidetes were more abundant in musk deer consuming a high-starch and high-protein diet during the summer; meanwhile, Firmicutes were more abundant in musk deer consuming a high-fiber diet during the winter [32, 41, 42]. Except for various leaves, the food of captive forest musk is mainly carrots and various grains. Compared with the Siberian musk deer, forest musk deer food contains more protein and fat, coupled with a lot of energy to avoid foraging and avoiding natural enemies, it is not difficult to understand that Bacteroides is higher in the forest musk deer.

In Siberian musk deer, the Firmicutes/Bacteroidetes ratio (F/B ratio: 6.6173-10.113) was significantly higher than that of forest musk (F/B ratio: 1.3843-2.2011). This may be related to the feeding habits of 
these animals, and these seasonal changes could increase energy efficiency during the winter $[10,43]$. Compared to forest musk deer, the Siberian musk deer has a broader diet, a relatively high living altitude, and a colder climate that requires more energy $[2,44]$. Recent research reports $[45,46]$ have confirmed that the ratio of Firmicutes and Bacteroidetes is related to fat deposition. Although these bacteria can promote fat deposition, when the abundance of Firmicutes increases, it can promote fat. The effect of deposition is more pronounced. Other studies [47] have shown that the genes of intestinal microorganisms with high F/B ratios are rich in encoding lysing polysaccharide start enzymes and eight glycosidic bond hydrolase families involved in sugar metabolism. And the residues of nutrients in mice with a high F/B ratio were significantly lower than those in lean mice. These results indicate that intestinal microorganisms with a high $\mathrm{F} / \mathrm{B}$ ratio have higher fermentation efficiency and obtain more energy from food, thereby promoting fat deposition. Therefore, it is believed that Firmicutes can help the body to absorb more energy from food. In our experiments involving siberian musk deer, life in the wild, winter cold and the shortage of food, a lot of energy on the one hand to find food, avoid predators, on the one hand, but also consume energy to maintain body temperature. So the Firmicutes in their gut microbiota can help them draw energy from food as much as possible to maintain the body's needs. Spirochaetae were only found in the FMD group. This may be related to feeding conditions and animal habits. In this study, Siberian musk deer lived in a wide range and had a primitive diet, while forest musk deer were raised artificially in captivity and fed by breeders. Similar phenomena have been found in previous studies on sika deer [30] and human living environment [48] -- different living environment makes spirochaetae only appear in intestinal flora of a certain living environment. In addition, studies have shown that animals fed diets rich in fungi, vitamin $\mathrm{C}$ and dietary fiber have lower levels of spirochaetae in gut microbiota [49], which is in line with our experimental animal Siberian musk deer similar, but need further validation.

The PCoA analyses were also showing the distinct separations and reciprocal relationships among musk deer from different areas, it indicated that the composition and structure of the bacterial community were significantly different between forest and siberian musk deer. The season factors would also not be negligible effect factors on musk deer gut microbiota. For forest musk deer, have 8 phyla bacteria were significantly different, and this result is similar to that of Hu et al $[18,26,27]$. However, the SMD group was less affected by seasons, and only one phylum bacteria (Tenericutes) significant difference. Phylum Tenericutes, class Mollicutes were rarely found in the gastrointestinal tract before and then were identified for the first time in wild chimpanzees, and Tenericutes also were discovered in herbivorous mammals [50, 51]. Consistent with the research results of gut microbiota of wild sika deer [30], which was the relative abundance of Tenericutes higher than Proteobacteria. Hence, Tenericutes should be valued for their special gut microbiota function in the musk deer. As we all know, the gut microbiota can affect the health and metabolism of the host. Among them, the large number of microorganisms and the complexity of the genomes associated with the microbial community limit the study of the metabolic profile of the bacterial community $[23,52,53]$. PICRUSt analysis enables robust assessments regarding the impact of environmental factors and biological processes on the composition of microbial communities. Microbial functional analysis showed that the most abundant biological pathways in all samples were translation, 
amino acid metabolism, carbohydrate metabolism, replication and repair, and membrane transport; this agrees with previous studies [54- 56]. In the SMD group, some pathways differed between the two seasons. Especially in the disease, the results show a variety of diseases was significantly enriched in the summer sample, the results of this follow-up study provides a guideline. PICRUSt analysis does have limitations, as other pathways, such as some disease, were identified as significant but are not relevant to microbial communities of musk deer. Transcriptomic and metabolomic analyses would be more applicable to determining bacterial functions.

This is the first characterization of the gut microbiota in SMD, and it is also the first time to compare the structure of the gut microbiota in FMD and SMD. We show that that musk deer gut microbiota is complex and taxonomically plastic, and it varies by species of musk deer. Microbiota composition changes seasonally, likely due to changes in environmental conditions and diet. Limitations of this study include the sample size collected was relatively small, the inability to control for potentially important factors, such as weather and the lack of species-level resolution via 16S rRNA tagged sequencing. Shotgun metagenomic sequencing would provide additional insight into the gut microbial community in musk deer. In light of the rapid development of agriculture and technology, as well as the urgent need for wildlife conservation, further research on the gut microbiota of musk deer is warranted.

\section{Conclusions}

We characterized the seasonal variation in the gastrointestinal microbiota of Siberian musk deer and forest musk deer using high-throughput sequencing of the 16S rRNA gene in fecal samples. The results show that the composition and diversity gut microbiota of these two musk deer has significant differences. The Firmicutes and Bacteroidetes were the predominant phyla in both species, their relative abundance was significantly different with species and seasonal factors. In addition, the microbial adiversity is higher in summer than winter, and determine seasonal variations in the microbial community composition of Siberian musk deer. This study is the first to analyze the composition of the gut microbiota in Siberian musk deer, and these results provide a foundation for improving feeding for musk deer populations and further studies investigating the environmental impact of musk deer.

\section{Methods}

\section{Animals and Sample Collection}

Four healthy adult Siberian musk deer were sampled from the musk-deer breeding center in Mongolia (Gachuurt Village, Ulaanbaatar; $47^{\circ} 55^{\prime} \mathrm{N} 107^{\circ} 09^{\prime} \mathrm{E}$ ). The animals used in this experiment were captured from the wild status by the staff of the Mongolian musk-deer breeding center, which is the partner of the project, and kept in pens within a certain range of breeding. The wild habit of the Siberian musk deer is protected to a certain extent, through a wider range of captive breeding (each musk deer is raised in pen of $20 \mathrm{~m} \times 40 \mathrm{~m}$ ) and avoiding the disturbance of the breeders as much as possible (Fig S7). The altitude of the breeding center is about $1783 \mathrm{~m}$, and the annual average precipitation is $200-400 \mathrm{~mm}$. The average 
summer temperature is $20-30{ }^{\circ} \mathrm{C}$, which is hot and cool. The average temperature in winter is about - 30 ${ }^{\circ} \mathrm{C}$, and the lowest temperature is $-40^{\circ} \mathrm{C}$. The siberian musk deer food partly from the leaves of plants grown in the fence, another part of the feed is mainly supplied by keepers such as Cetrarian lichens and mushrooms (Fig S7). They spend 4-5 hours and about 9 hours in summer days and winter days respectively, to feed with a repetition of 5-8 times a day during the dusk and dawn. Stomach filled with food weighs $400-600 \mathrm{gr}$, on average it is $520 \mathrm{gr}$. In July, their diet is $80 \%$ fresh green plants and $20 \%$ moss and lichens. In December, their diet is $90 \%$ Cetrarian lichens (e.g., Cetrariahivaeis, Cetrariaislanica, Cetrariacucuetata) and $10 \%$ green moss. They also consume pine nuts, barberry leaves, pine, and spruce (Table 1; Table S4). During the summer Siberian musk deer eats $2 \mathrm{~kg}$ of a green plant, $0.6 \mathrm{~kg}$ moss lichens or moss, in winter $3.7 \mathrm{~kg}$ lichens, $0.4 \mathrm{~kg}$ moss per day. According to the research Musk deer drinks $100 \mathrm{ml}$ of water for once, 3-4 times a day.

Ten forest musk deer were sampled from the Inner Mongolia Ulantasb League in China $\left(40^{\circ} 42^{\prime} \mathrm{N}\right.$ $113^{\circ} 26^{\prime} \mathrm{E}$ ). The average altitude is 1400 meters and the average rainfall is $400 \mathrm{~mm}$. The hottest month is July, the average temperature is $20.4^{\circ} \mathrm{C}$, the highest temperature is $36.5^{\circ} \mathrm{C}$, the average temperature in winter is $-13.5^{\circ} \mathrm{C}$, and the lowest temperature is $-37.5^{\circ} \mathrm{C}$. Forest musk deer diet consisted mainly of leaves (e.g., mulberry leaves (Morus alba), elm leaves (Ulmus pumila), broussonetia papyrifera leaves) and seasonal vegetables (e.g., pumpkin, carrots, and corn). They were fed with fresh leaves in the summer and dried leaves in the winter. Under captive conditions, due to changes in environmental conditions, the type of feeding is greatly restricted, so a proper supply of local vegetables and minerals is essential. Each kilogram of leaves contains $250 \mathrm{mg}$ of carotene, $1200 \mathrm{mg}$ of calcium, $320 \mathrm{mg}$ of iron, and also contains copper, zinc, phosphorus, manganese and other trace elements and vitamins. The content of crude protein in the dry matter of the foliage feed is $17 \%$ to $22 \%$. The content was up to $36 \%$ in June, and gradually decreased after July, and fell to $12 \%$ in November.

Ten fecal samples were collected from forest musk deer in both summer (July, FS1-FS10) and winter (December, FW1-FW10). 4 fecal samples were collected from Siberian musk deer in both summer (July, SS6,SS8-SS10) and winter (December, SW1-SW4). Musk deer usually defecate in the early morning (around 5-6 o 'clock) with fixed defecation sites. Fresh fecal samples were collected with a sterile disposable glove to avoid human contamination. Samples were stored in sterile centrifuge tubes, sealed, and placed on liquid nitrogen for transportation to the laboratory. Samples were then stored at $-80^{\circ} \mathrm{C}$ until DNA extraction.

All experiment and animal care activities conducted according to the animal ethics guidelines of the Bioethics Committee of the College of Veterinary Medicine in Inner Mongolia Agricultural University $(12150000460029509 \mathrm{~N})$ and Institute of Traditional Medicine and Technology, Ulaanbaatar, Mongolia. And we got a permit of CITES management authority of Mongolia in 2018.

\section{DNA Extraction}

Bacterial DNA was extracted using the QIAamp ${ }^{\circledR}$ DNA Stool Mini Kit (Qiagen, Hilden, Germany) according to the manufacturer's instructions. Samples were crushed on dry ice before extraction. The DNA 
concentration was measured using a NanoDrop 2000 Spectrophotometer and confirmed by $1.0 \%$ agarose gel electrophoresis. The concentration and purity of DNA were tested using the Qubit dsDNA HS Assay Kit (Life Technologies, Carlsbad, CA, USA). Extracted DNA was stored at $-80^{\circ} \mathrm{C}$ until PCR.

\section{PCR Amplification and 16S rRNA Gene Sequencing}

The hypervariable V3-V4 region of the 16S rRNA gene was amplified using the universal bacterial PCR primers 338F (5'-ACTCCTACGGGAGGCAGCA-3') and 806R (5'-GGACTACHVGGGTWTCTAAT-3') [57]. The total reaction volume was $20 \mu \mathrm{L}$ mixture, and consisted of: $4 \mu \mathrm{L} 5 \times$ FastPfu Buffer, $2 \mu \mathrm{L}$ dNTPs $(2.5 \mathrm{mM})$, $0.8 \mu \mathrm{L}$ Forward Primer $(5 \mu \mathrm{M}), 0.8 \mu \mathrm{L}$ Reverse Primer $(5 \mu \mathrm{M}), 0.4 \mu \mathrm{L}$ FastPfu Polymerase, $0.2 \mu \mathrm{L}$ BSA, $10 \mathrm{ng}$ Template DNA, and the remaining volume was $\mathrm{ddH}_{2} \mathrm{O}$. The PCR conditions were: 3 min at $95^{\circ} \mathrm{C}$ for initial denaturation; followed by 27 cycles of $95^{\circ} \mathrm{C}$ for $30 \mathrm{~s}, 55^{\circ} \mathrm{C}$ for $30 \mathrm{~s}$ and $72^{\circ} \mathrm{C}$ for $45 \mathrm{~s}$, and; a final extension step for $10 \mathrm{~min}$ at $72^{\circ} \mathrm{C}$. PCR products were extracted from $2 \%$ agarose gels, purified with the AxyPrep DNA Gel Extraction Kit (Axygen Biosciences, Union City, CA, USA), and quantified using the QuantiFluor ${ }^{\text {TM }}$ ST Kit (Promega, USA). Samples were sequenced on a MiSeq (300 bp paired-end reads) at the Majorbio Bio-pharm Technology Co., Ltd (Shanghai, China).

\section{Bioinformatics and Statistical Analyses}

Sequence assembly and quality filtering were performed using FLASH (version 1.2.11; https://ccb.jhu.edu/software/FLASH/index.shtml) and QIIME (version 1.9.1; http://qiime.org/install/index.html), respectively. The quality control method was: 1) filter bases with a tail mass value $<20$ and set a $50 \mathrm{bp}$ window. If the average mass value in the window was lower than 20 , the back base was truncated from the window. Reads less than 50 bp were removed; 2) Based on the overlap between the PE reads, the reads were merged with a minimum overlap length of $10 \mathrm{bp} ; 3$ ) The maximum mismatch ratio allowed by the overlap area of the splicing sequence was $0.2 ; 4)$ Sequences were assigned to samples using barcoded primers. Operational taxonomic units (OTUs) were picked using Usearch (version 7.0.1090, http://drive5.com/uparse/).

Taxonomy was assigned to the OTUs using the Ribosomal Database Project (RDP) classifier (version 2.11, http://sourceforge.net/projects/rdp-classifier/) against the Silva 132 (Release132 http://www.arbsilva.de) database with a confidence threshold of 0.97. Alpha diversity indices, including Chao, ACE, Shannon, Simpson, and Good's Coverage, as well as beta diversity analysis, were performed in Mothur (version v.1.30.2, http://www.mothur.org/wiki/Schloss_SOP\#Alpha_diversity) and visualized using R (version 3.5.0). Wilcoxon rank-sum tests were used to determine statistically significant differences in alpha diversity and taxa relative abundance. Venn diagrams and pie charts were used to visualize shared OTUs, which were defined as the core microbiome, using the Venn Diagram package in R. Heatmaps were generated using Bray-Curtis dissimilarity with average linkage hierarchical clustering using the vegan package in R. Principal co-ordinates analysis (PCOA) ordination based on Bray- Curtis dissimilarities and an analysis of similarity (ANOSIM) test were used to determine the composition differences and the significance of community compositional differences among groups. Perform PCoA Statistical analysis 
and plotting used the vegan package in R. Differences between groups were determined using KruskalWallis $\mathrm{H}$-tests using the stats package in $\mathrm{R}$ and the SciPy package in Python (version 2.6.4). Linear discriminant analysis effect size (LEfse) analysis was conducted to indicate the significant factors using the online Galaxy workflow framework (http://huttenhower.sph.harvard.edu/galaxy/) and the cutoff value for the linear discriminant analysis score was set to the default level of 4.0 [58]. PICRUSt (Phylogenetic Investigation of Communities by Reconstruction of Unobserved States, http://picrust.github.io/picrust/index.html) was used to predict the functional gene content of microorganisms (Langille MG et al., 2013)[59]. The predicted function is precalculated for genes in the Kyoto Encyclopedia of Genes and Genomes (KEGG, http://www.genome.jp/kegg/) database were used to predict gene function. STAMP analysis was used to assess functional richness between groups. Welch's t-test was used for STAMP (version v2.1.3) software for differential analysis. Differences were considered as statistically significant when $P<0.05$.

\section{Abbreviations}

SMD: Siberian musk deer (Moschus moschiferus)

FMD: forest musk deer (Moschus berezovskii)

FW: forest musk deer in the winter

FS: forest musk deer in the summer

SS: Siberian musk deer in the summer

SW: Siberian musk deer in the winter

OTUs: Operational taxonomic units

PCoA: Principal co-ordinates analysis

ANOSIM: an analysis of similarity test

LEfse: Linear discriminant analysis effect size analysis

KEGG: the Kyoto Encyclopedia of Genes and Genomes database

\section{Declarations}

\section{Ethics approval and consent to participate}

All experiment and animal care activities conducted according to the animal ethics guidelines of the Bioethics Committee of the College of Veterinary Medicine in Inner Mongolia Agricultural University 
$(12150000460029509 N)$ and Institute of Traditional Medicine and Technology in Mongolia, Ulaanbaatar. And we got a permit of CITES management authority of Mongolia in 2018.

\section{Consent for publication}

Not applicable.

\section{Availability of data and materials}

The raw sequences obtained in this study can be found in the NCBI Sequence Read Archive with the accession number: SRP198443.

\section{Competing interests}

All authors and funding bodies declare that they have no competing interests.

\section{Funding}

This work was supported by the National International Scientific and Technological Cooperation Project (Grant number is 2016YFE0116500).

\section{Author contributions}

SH and CC designed and led the project, and revised the manuscript; RNS contributed to complete all experiments and analysis of experimental data and wrote the manuscript. MD, ME and BL participated in the sample collection and analysis of experimental data. All authors have read and approved the final manuscript.

\section{Acknowledgments}

We would like to thank the native English speaking scientists of Elixigen Company (Huntington Beach, California) for editing our manuscript. And special thanks to all the breeders of musk deer in China and Mongolia.

\section{Authors' information}

Rina Su: sliya@emails.imau.edu.cn

Menggen Dalai: Prof., mengendalai@sina.com

Myagmarsuren Erdenedalai : mygaa_e09@yahoo.com

Batkhuu L: Prof., enshibat@yahoo.com

Chimedragchaa Chi: Prof., ch.chimedragchaa@yahoo.com 
Surong Hasi: Prof., surong@imau.edu.cn

\section{Additional file}

\section{Additional file 1:}

Supplementary material contains Fig S1-S7 and Table S1-S4 with detailed results for the Figures presented in the main manuscript.

\section{References}

1. Chimedragchaa $\mathrm{CH}$, Mengen Dalai, Batkhuu L, Surong Hasi, Dejidmaa B, Erenechimeg $\mathrm{CH}$, et al. Musk deer (Moschus moschiferus Linnaeus.,1758) and study of musk. Ulaanbaater: Mongolia; 2019, ISBN 978-9919-21-868-5.

2. Homes V. No Licence to Kill: the Population and Harvest of Musk Deer and Trade in Musk in the Russian Federation and Mongolia. TRAFFIC, Europe, 2004.

3. Bao, Y, Sun, J.M, Zhang, H . Moschus moschiferus resource status and protective breeding measures. Jilin Journal of Traditional Chinese Medicine. 2015; 35: 703-706.

4. Tsendjav D, Bujinkhand Ch. Present and Future of Musk Deer (Moschus moschiferus L, 1758). Proceedings of the Institute of General and Experimental Biology of the Mongolian Academy of Sciences. 2000; 22: 91-94.

5. Consideration of Proposals for Amendment of Appendices I and II. Proposal 11.29. Eleventh meeting of the Conference of the Parties, Gigiri, 2002.

6. Togtokhbayar D, Buyantsog B, Monkhtsog B. Information on Musk Deer Distribution. Proceedings of the Institute of General and Experimental Biology of the Mongolian Academy of Sciences. 2000; 22: 245-246.

7. Ezenwa VO, Gerardo NM, Inouye DW, Medina M, Xavier JB. Microbiology. Animal behavior and the microbiome. Science. 2012;338;6104:198-199.

8. Heys C, Cheaib B, Busetti A, Kazlauskaite R, Maier L, Sloan WT, et al. Neutral Processes Dominate Microbial Community Assembly in Atlantic Salmon, Salmo salar. Appl Environ Microbiol. 2020 ; 86(8):e02283-19. doi: 10.1128/AEM.02283-19. PMID: 32033945; PMCID: PMC7117918.

9. Carding S, Verbeke K, Vipond DT, Corfe BM, Owen LJ. Dysbiosis of the gut microbiota in disease. Microb Ecol Health Dis. 2015;26:26191. doi: 10.3402/mehd.v26.26191. PMID: 25651997; PMCID: PMC4315779.

10. Chevalier C, Stojanović O, Colin DJ, Suarez-Zamorano N, Tarallo V, Veyrat-Durebex C, et al. Gut Microbiota Orchestrates Energy Homeostasis during Cold. Cell. 2015;16(6):1360-74. doi: 10.1016/j.cell.2015.11.004. PMID: 26638070.

11. Roeselers G, Mittge EK, Stephens WZ, Parichy DM, Cavanaugh CM, Guillemin K, et al. Evidence for a core gut microbiota in the zebrafish. ISME J. $2011 ; 5(10): 1595-608$. doi: 10.1038/ismej.2011.38. 
Epub 2011 Apr 7. PMID: 21472014; PMCID: PMC3176511.

12. Li H, Qu J, Li T, Yao M, Li J, Li X. Gut microbiota may predict host divergence time during Glires evolution. FEMS Microbiol Ecol. 2017;93:3. doi: 10.1093/femsec/fix009. PMID: 28137765.

13. Holman DB, Chénier MR. Temporal changes and the effect of subtherapeutic concentrations of antibiotics in the gut microbiota of swine. FEMS Microbiol Ecol. 2014;90(3):599-608. doi:

10.1111/1574-6941.12419. Epub 2014 Oct 2. PMID: 25187398.

14. Clark A, Sallé G, Ballan V, Reigner F, Meynadier A, Cortet J, et al. Strongyle Infection and Gut Microbiota: Profiling of Resistant and Susceptible Horses Over a Grazing Season. Front Physiol. 2018 ;9:272. doi: 10.3389/fphys.2018.00272. PMID: 29618989; PMCID: PMC5871743.

15. Maurice CF, Knowles SC, Ladau J, Pollard KS, Fenton A, Pedersen AB, et al. Marked seasonal variation in the wild mouse gut microbiota. ISME J. 2015;9(11):2423-34. doi: 10.1038/ismej.2015.53. Epub 2015 May 29. PMID: 26023870; PMCID: PMC4611506.

16. Wu Q, Wang X, Ding Y, Hu Y, Nie Y, Wei W, et al. Seasonal variation in nutrient utilization shapes gut microbiome structure and function in wild giant pandas. Proc Biol Sci. 2017;284(1862):20170955. doi: 10.1098/rspb.2017.0955. PMID: 28904137; PMCID: PMC5597826.

17. Smits SA, Leach J, Sonnenburg ED, Gonzalez CG, Lichtman JS, Reid G, et al. Seasonal cycling in the gut microbiome of the Hadza hunter-gatherers of Tanzania. Science. 2017; 357 (6353): 802-806. doi: 10.1126/science.aan4834. PMID: 28839072; PMCID: PMC5891123.

18. Hu X, Liu G, Li Y, Wei Y, Lin S, Liu S, et al. High-Throughput Analysis Reveals Seasonal Variation of the Gut Microbiota Composition Within Forest Musk Deer (Moschus berezovskii). Front Microbiol. 2018;9:1674. doi: 10.3389/fmicb.2018.01674. PMID: 30093891; PMCID: PMC6070636.

19. Brugère JF, Mihajlovski A, Missaoui M, Peyret $P$. Tools for stools: the challenge of assessing human intestinal microbiota using molecular diagnostics. Expert Rev Mol Diagn. 2009;9(4):353-65. doi: 10.1586/erm.09.16. PMID: 19435456.

20. Yan M, Yan QG, Yang GY. The mass diseases of captive musk deer. J. Econ. Anim. 2016;20: 112117.

21. Wang YQ, Meng XX. The current status and disease occurrence of captive forest musk deer (Moschus berezovskii) in Shaanxi Province. Chinese Journal of Ecology. 2014; 33: 2720-2726.

22. Wei H, Dong L, Wang T, Zhang M, Hua W, Zhang C,et al. Structural shifts of gut microbiota as surrogate endpoints for monitoring host health changes induced by carcinogen exposure. FEMS Microbiol Ecol. 2010;73(3):577-86.

23. Winter SE, Thiennimitr P, Winter MG, Butler BP, Huseby DL, Crawford RW, et al. Gut inflammation provides a respiratory electron acceptor for Salmonella. Nature. $2010 ; 467(7314): 426-9$. doi: 10.1038/nature09415. PMID: 20864996; PMCID: PMC2946174.

24. Liu C, Wu H, Liu S, Chai S, Meng Q, Zhou Z. Dynamic Alterations in Yak Rumen Bacteria Community and Metabolome Characteristics in Response to Feed Type. Front Microbiol. 2019;10:1116. doi: 10.3389/fmicb.2019.01116. PMID: 31191470; PMCID: PMC6538947. 
25. Sonnenburg ED, Smits SA, Tikhonov M, Higginbottom SK, Wingreen NS, Sonnenburg JL. Diet-induced extinctions in the gut microbiota compound over generations. Nature. 2016;529(7585):212-5. doi: 10.1038/nature16504. PMID: 26762459; PMCID: PMC4850918.

26. Hu X, Liu G, Shafer ABA, Wei Y, Zhou J, Lin S, et al. Comparative Analysis of the Gut Microbial Communities in Forest and Alpine Musk Deer Using High-Throughput Sequencing. Front Microbiol. 2017;8:572.

27. Li Y, Hu X, Yang S, Zhou J, Zhang T, Qi L, et al. Comparative Analysis of the Gut Microbiota Composition between Captive and Wild Forest Musk Deer. Front Microbiol. 2017 ;8:1705. doi: 10.3389/fmicb.2017.01705. PMID: 28928728; PMCID: PMC5591822.

28. Henderson G, Cox F, Ganesh S, Jonker A, Young W. Global Rumen Census Collaborators, Janssen PH. Rumen microbial community composition varies with diet and host, but a core microbiome is found across a wide geographical range. Sci Rep. 2015;5:14567.

29. Delgado ML, Singh P, Funk JA, Moore JA, Cannell EM, Kanesfsky J, et al. Intestinal Microbial Community Dynamics of White-Tailed Deer (Odocoileus virginianus) in an Agroecosystem. Microb Ecol. $2017 ; 74(2): 496-506$.

30. Guan Y, Yang H, Han S, Feng L, Wang T, Ge J. Correction to: Comparison of the gut microbiota composition between wild and captive sika deer (Cervus nippon hortulorum) from feces by highthroughput sequencing. AMB Express. 2018;8(1):15. doi: 10.1186/s13568-018-0535-1. Erratum for: AMB Express. 2017 Nov 23;7(1):212. PMID: 29404873; PMCID: PMC5799092.

31. Ley RE, Hamady M, Lozupone C, Turnbaugh PJ, Ramey RR, Bircher JS, et al. Evolution of mammals and their gut microbes. Science. 2008;320(5883):1647-51. doi: 10.1126/science.1155725. Epub 2008 May 22. Erratum in: Science. 2008 Nov 21;322(5905):1188. PMID: 18497261; PMCID: PMC2649005.

32. Fernando SC, Purvis HT 2nd, Najar FZ, Sukharnikov LO, Krehbiel CR, Nagaraja TG, et al. Rumen microbial population dynamics during adaptation to a high-grain diet. Appl Environ Microbiol. 2010;76(22):7482-90. doi: 10.1128/AEM.00388-10. Epub 2010 Sep 17. PMID: 20851965; PMCID: PMC2976194.

33. Waite DW, Taylor MW. Characterizing the avian gut microbiota: membership, driving influences, and potential function. Front Microbiol. 2014;5:223. doi: 10.3389/fmicb.2014.00223. PMID: 24904538; PMCID: PMC4032936.

34. Nuriel-Ohayon M, Neuman $\mathrm{H}$, Koren O. Microbial Changes during Pregnancy, Birth, and Infancy. Front Microbiol. 2016;7:1031. doi: 10.3389/fmicb.2016.01031. PMID: 27471494; PMCID: PMC4943946.

35. Krause DO, Denman SE, Mackie RI, Morrison M, Rae AL, Attwood GT, et al. Opportunities to improve fiber degradation in the rumen: microbiology, ecology, and genomics. FEMS Microbiol Rev. 2003c;27(5):663-93. doi: 10.1016/S0168-6445(03)00072-X. PMID: 14638418.

36. Davenport ER, Mizrahi-Man O, Michelini K, Barreiro LB, Ober C, Gilad Y. Seasonal variation in human gut microbiome composition. PLoS One. 2014;9(3):e90731. doi: 10.1371/journal.pone.0090731. PMID: 24618913; PMCID: PMC3949691. 
37. Scott KP, Gratz SW, Sheridan PO, Flint HJ, Duncan SH. The influence of diet on the gut microbiota. Pharmacol Res. 2013;69(1):52-60. doi: 10.1016/j.phrs.2012.10.020. Epub 2012 Nov 9. PMID: 23147033.

38. Xiao S, Fei N, Pang X, Shen J, Wang L, Zhang B, et al. A gut microbiota-targeted dietary intervention for amelioration of chronic inflammation underlying metabolic syndrome. FEMS Microbiol Ecol. 2014;87(2):357-67. doi: 10.1111/1574-6941.12228. Epub 2013 Oct 21. PMID: 24117923; PMCID: PMC4255291.

39. Malmuthuge N, Liang G, Griebel PJ, Guan LL. Taxonomic and Functional Compositions of the Small Intestinal Microbiome in Neonatal Calves Provide a Framework for Understanding Early Life Gut Health. Appl Environ Microbiol. 2019;85(6):e02534-18. doi: 10.1128/AEM.02534-18. PMID: 30658973; PMCID: PMC6414372.

40. Belanche A, Doreau M, Edwards JE, Moorby JM, Pinloche E, Newbold CJ. Shifts in the rumen microbiota due to the type of carbohydrate and level of protein ingested by dairy cattle are associated with changes in rumen fermentation. J Nutr. 2012;142(9):1684-92. doi: 10.3945/jn.112.159574. Epub 2012 Jul 25. PMID: 22833657.

41. Li ZP, Liu HL, Li GY, Bao K, Wang KY, Xu C, et al. Molecular diversity of rumen bacterial communities from tannin-rich and fiber-rich forage fed domestic Sika deer (Cervus nippon) in China. BMC Microbiol. 2013;13:151. doi: 10.1186/1471-2180-13-151. PMID: 23834656; PMCID: PMC3723558.

42. Agans R, Gordon A, Kramer DL, Perez-Burillo S, Rufián-Henares JA, Paliy O. Dietary Fatty Acids Sustain the Growth of the Human Gut Microbiota. Appl Environ Microbiol. 2018;84(21):e01525-18. doi: 10.1128/AEM.01525-18. PMID: 30242004; PMCID: PMC6193386.

43. Carding S, Verbeke K, Vipond DT, Corfe BM, Owen LJ. Dysbiosis of the gut microbiota in disease. Microb Ecol Health Dis. 2015;26:26191. doi: 10.3402/mehd.v26.26191. PMID: 25651997; PMCID: PMC4315779.

44. Yi L, Dalai M, Su R, Lin W, Chimedtseren C, Hasi S,et al. Whole-genome sequencing of wild Siberian musk deer (Moschus moschiferus) provides insights into its genetic features. BMC Genomics. 2020 ;21(1):108. doi: 10.1186/s12864-020-6495-2. PMID: 32005147; PMCID: PMC6995116.

45. Indiani CMDSP, Rizzardi KF, Castelo PM, Ferraz LFC, Darrieux M, Parisotto TM. Childhood Obesity and Firmicutes/Bacteroidetes Ratio in the Gut Microbiota: A Systematic Review. Child Obes. 2018 ;14(8):501-509. doi: 10.1089/chi.2018.0040. Epub 2018 Sep 5. PMID: 30183336.

46. Koliada A, Syzenko G, Moseiko V, Budovska L, Puchkov K, Perederiy V, et al. Association between body mass index and Firmicutes/Bacteroidetes ratio in an adult Ukrainian population. BMC Microbiol. 2017;17(1):120. doi: 10.1186/s12866-017-1027-1. PMID: 28532414; PMCID: PMC5440985.

47. Ley RE, Turnbaugh PJ, Klein S, Gordon JI. Microbial ecology: human gut microbes associated with obesity. Nature. 2006;444(7122):1022-3. doi: 10.1038/4441022a. PMID: 17183309.

48. Angelakis E, Bachar D, Yasir M, Musso D, Djossou F, Gaborit B, et al. Treponema species enrich the gut microbiota of traditional rural populations but are absent from urban individuals. New Microbes 
New Infect. 2018;27:14-21. doi: 10.1016/j.nmni.2018.10.009. PMID: 30555706; PMCID: PMC6276622.

49. Nuli R, Cai J, Kadeer A, Zhang Y, Mohemaiti P. Integrative Analysis Toward Different Glucose Tolerance-Related Gut Microbiota and Diet. Front Endocrinol (Lausanne). 2019;10:295. doi: 10.3389/fendo.2019.00295. PMID: 31191448; PMCID: PMC6546033.

50. Szekely BA, Singh J, Marsh TL, Hagedorn C, Werre SR, Kaur T. Fecal bacterial diversity of humanhabituated wild chimpanzees (Pan troglodytes schweinfurthii) at Mahale Mountains National Park, Western Tanzania. Am J Primatol. 2010 ;72(7):566-74. doi: 10.1002/ajp.20809. PMID: 20146237.

51. JJami E, Mizrahi I. Composition and similarity of bovine rumen microbiota across individual animals. PLoS One. 2012;7(3):e33306. doi: 10.1371/journal.pone.0033306. Epub 2012 Mar 14. PMID: 22432013; PMCID: PMC3303817.

52. Goodrich JK, Waters JL, Poole AC, Sutter JL, Koren O, Blekhman R, et al. Human genetics shape the gut microbiome. Cell. 2014;159(4):789-99. doi: 10.1016/j.cell.2014.09.053. PMID: 25417156; PMCID: PMC4255478.

53. Mukhopadhya I, Hansen R, El-Omar EM, Hold GL. IBD-what role do Proteobacteria play? Nat Rev Gastroenterol Hepatol. 2012 ;9(4):219-30. doi: 10.1038/nrgastro.2012.14. PMID: 22349170.

54. Arumugam M, Raes J, Pelletier E, Le Paslier D, Yamada T, Mende DR,et al. Enterotypes of the human gut microbiome. Nature. 2011 May 12;473(7346):174-80. doi: 10.1038/nature09944. Epub 2011 Apr 20. Erratum in: Nature. 2011;474(7353):666. Erratum in: Nature. 2014 Feb 27;506(7489):516. PMID: $21508958 ;$ PMCID: PMC3728647.

55. Ridaura VK, Faith JJ, Rey FE, Cheng J, Duncan AE, Kau AL, et al. Gut microbiota from twins discordant for obesity modulate metabolism in mice. Science. 2013; 341(6150):1241214. doi: 10.1126/science.1241214. PMID: 24009397; PMCID: PMC3829625.

56. Lu K, Abo RP, Schlieper KA, Graffam ME, Levine S, Wishnok JS,et al. Arsenic exposure perturbs the gut microbiome and its metabolic profile in mice: an integrated metagenomics and metabolomics analysis. Environ Health Perspect. 2014;122(3):284-91. doi: 10.1289/ehp.1307429. Epub 2014 Jan 10. PMID: 24413286; PMCID: PMC3948040.

57. Yu Z, Morrison M. Comparisons of different hypervariable regions of rrs genes for use in fingerprinting of microbial communities by PCR-denaturing gradient gel electrophoresis. Appl Environ Microbiol. 2004; 70: 800-4806.

58. Segata N, Izard J, Waldron L, Gevers D, Miropolsky L, Garrett WS, et al. Metagenomic biomarker discovery and explanation. Genome Biol. 2011;12(6):R60. doi: 10.1186/gb-2011-12-6-r60. PMID: 21702898; PMCID: PMC3218848.

59. Langille MG, Zaneveld J, Caporaso JG, McDonald D, Knights D, Reyes JA, et al. Predictive functional profiling of microbial communities using 16S rRNA marker gene sequences. Nat Biotechnol. 2013;31(9):814-21. doi: 10.1038/nbt.2676. Epub 2013 Aug 25. PMID: 23975157; PMCID: PMC3819121. 


\section{Tables}

Table 1. Detailed characteristics of four population groups in this study.FW: forest musk deer in the winter; FS: forest musk deer in the summer; SS: Siberian musk deer in the summer; SW: Siberian musk deer in the winter.

\begin{tabular}{|c|c|c|c|c|c|c|}
\hline Species & $\begin{array}{l}\text { Population } \\
\text { group }\end{array}$ & Date & $\begin{array}{l}\text { Age } \\
\text { (yrs) }\end{array}$ & $\begin{array}{l}\text { Number } \\
\text { of } \\
\text { samples }\end{array}$ & $\begin{array}{l}\text { Geographic } \\
\text { distribution }\end{array}$ & Diet \\
\hline \multirow{2}{*}{$\begin{array}{l}\text { Siberian } \\
\text { musk deer } \\
\text { (SMD) }\end{array}$} & SW & $\begin{array}{l}\text { Dec } \\
2017\end{array}$ & $2-9$ & 4 & $\begin{array}{l}\text { Mongolia, } \\
\text { Ulaanbaatar }\end{array}$ & Abietoideae, Usnea,etc. \\
\hline & SS & $\begin{array}{l}\text { July } \\
2018\end{array}$ & $2-9$ & 4 & $\begin{array}{l}\text { Mongolia, } \\
\text { Ulaanbaatar }\end{array}$ & $\begin{array}{l}\text { Acerginnala, } \\
\text { Tricholomamongolicum, } \\
\text { etc. }\end{array}$ \\
\hline \multirow[t]{2}{*}{$\begin{array}{l}\text { Forest musk } \\
\text { deer (FMD) }\end{array}$} & FS & $\begin{array}{l}\text { Aug } \\
2018\end{array}$ & $\begin{array}{l}2.5- \\
5\end{array}$ & 10 & $\begin{array}{l}\text { China, Inner } \\
\text { Mongolia }\end{array}$ & $\begin{array}{l}\text { fresh leaves and } \\
\text { seasonal vegetables }\end{array}$ \\
\hline & FW & $\begin{array}{l}\text { Dec } \\
2018\end{array}$ & $\begin{array}{l}2.5- \\
5\end{array}$ & 10 & $\begin{array}{l}\text { China, Inner } \\
\text { Mongolia }\end{array}$ & $\begin{array}{l}\text { dry leaves and seasonal } \\
\text { vegetables }\end{array}$ \\
\hline
\end{tabular}

Table 2. Relative abundances (mean \pm SD) of bacterial phyla in summer and winter. Statistical significance was determined using Wilcoxon Rank-sum tests $(0.01<p \leq 0.05$ marked as *, $0.001<p \leq$ 0.01 marked as $* \star, p \leq 0.001$ marked as ${ }^{\star \star *}$ ). 


\begin{tabular}{|c|c|c|c|c|}
\hline Species & Species name & Summer (\%) & Winter (\%) & P-value \\
\hline \multirow[t]{15}{*}{ FMD } & Firmicutes $\rrbracket$ & $52 \pm 8.427$ & $64.03 \pm 11.69$ & 0.017 \\
\hline & 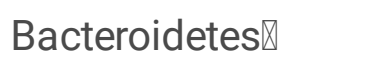 & $38.17 \pm 7.489$ & $29.09 \pm 8.978$ & 0.026 \\
\hline & Spirochaetes & $1.573 \pm 2.071$ & $3.41 \pm 6.899$ & 0.791 \\
\hline & Verrucomicrobia & $3.5 \pm 5.208$ & $1.05 \pm 1.788$ & 0.064 \\
\hline & Proteobacteria & $1.767 \pm 2.594$ & $0.68 \pm 1.156$ & 0.121 \\
\hline & Tenericutes & $0.79 \pm 0.533$ & $0.8 \pm 0.567$ & 0.909 \\
\hline & 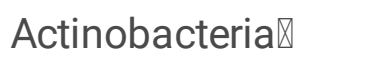 & $0.8 \pm 0.667$ & $0.36 \pm 0.391$ & 0.026 \\
\hline & 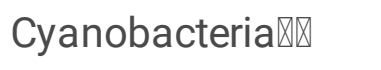 & $0.7 \pm 0.345$ & $0.26 \pm 0.226$ & $<0.01$ \\
\hline & 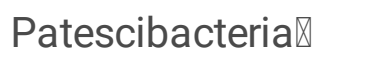 & $0.14 \pm 0.047$ & $0.14 \pm 0.337$ & 0.011 \\
\hline & Kiritimatiellaeota讴 & $0.19 \pm 0.374$ & $<0.01$ & 0.004 \\
\hline & Synergistetes & $0.16 \pm 0.374$ & $0.02 \pm 0.031$ & 0.235 \\
\hline & Lentisphaerae》 & $0.06 \pm 0.035$ & $0.04 \pm 0.084$ & 0.037 \\
\hline & Epsilonbacteraeota & $0.02 \pm 0.028$ & $0.04 \pm 0.045$ & 0.562 \\
\hline & Elusimicrobia胛 & $0.03 \pm 0.031$ & $<0.01$ & 0.004 \\
\hline & Deferribacteres & 0 & $<0.01$ & 0.078 \\
\hline \multirow[t]{13}{*}{ SMD } & Firmicutes & $84.57 \pm 6.305$ & $89.86 \pm 3.094$ & 0.312 \\
\hline & Bacteroidetes & $12.78 \pm 6.113$ & $8.89 \pm 2.302$ & 0.665 \\
\hline & Tenericutes $\bigotimes$ & $1.67 \pm 0.516$ & $0.35 \pm 0.238$ & 0.03 \\
\hline & Proteobacteria & $0.42 \pm 0.316$ & $0.72 \pm 1.018$ & 1 \\
\hline & Cyanobacteria & $0.25 \pm 0.091$ & $0.12 \pm 0.062$ & 0.11 \\
\hline & Actinobacteria & $0.19 \pm 0.154$ & $0.01 \pm 0.006$ & 0.065 \\
\hline & Elusimicrobia & $0.04 \pm 0.028$ & 0 & 0.069 \\
\hline & Patescibacteria & $0.03 \pm 0.041$ & $<0.01$ & 0.243 \\
\hline & Verrucomicrobia & $0.02 \pm 0.014$ & $<0.01$ & 0.18 \\
\hline & Epsilonbacteraeota & $<0.01$ & $<0.01$ & 0.619 \\
\hline & Lentisphaerae & $<0.01$ & $<0.01$ & 0.868 \\
\hline & Fusobacteria & $<0.01$ & $<0.01$ & 1 \\
\hline & Kiritimatiellaeota & $<0.01$ & 0 & 0.181 \\
\hline
\end{tabular}




\section{Figures}

A

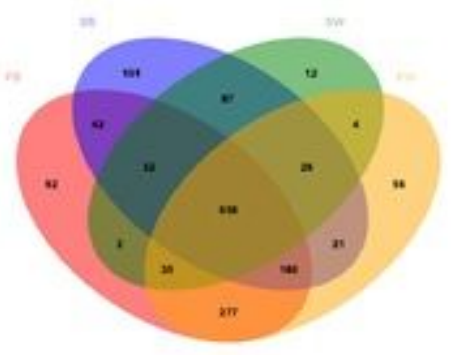

B
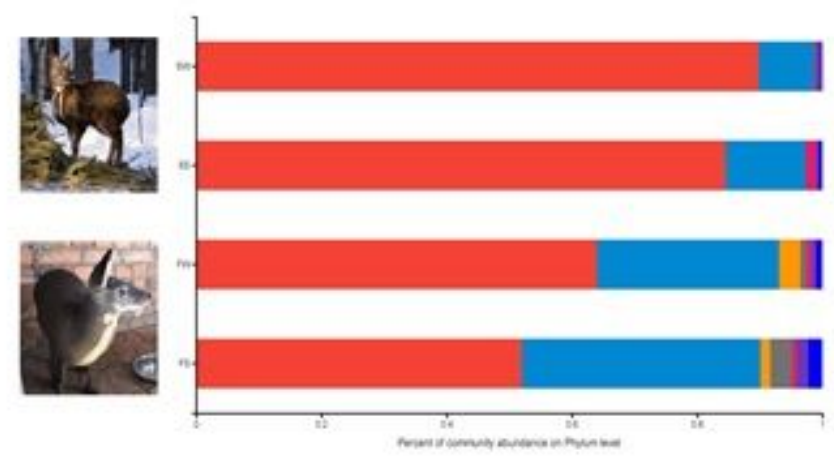

\section{Figure 1}

OTUs and bacterial taxa shared between the four groups. Venn diagrams show the number of OTUs shared by forest musk and Siberian musk deer sampled during two seasons (A). Bar charts showing the core bacterial taxa at the phylum level for each group: (B) . All taxa with relative abundances less than $1 \%$ were grouped as "Other taxa".FW: forest musk deer in the winter; FS: forest musk deer in the summer; SS: Siberian musk deer in the summer; SW: Siberian musk deer in the winter. 
A

Wicoxon rank-sum vest for shannon index

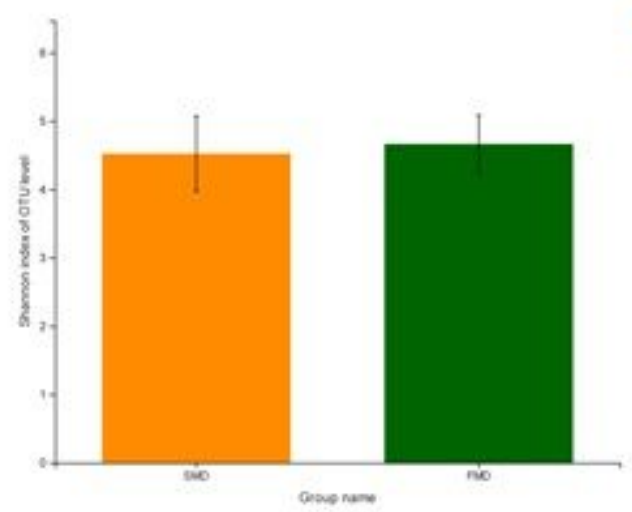

C

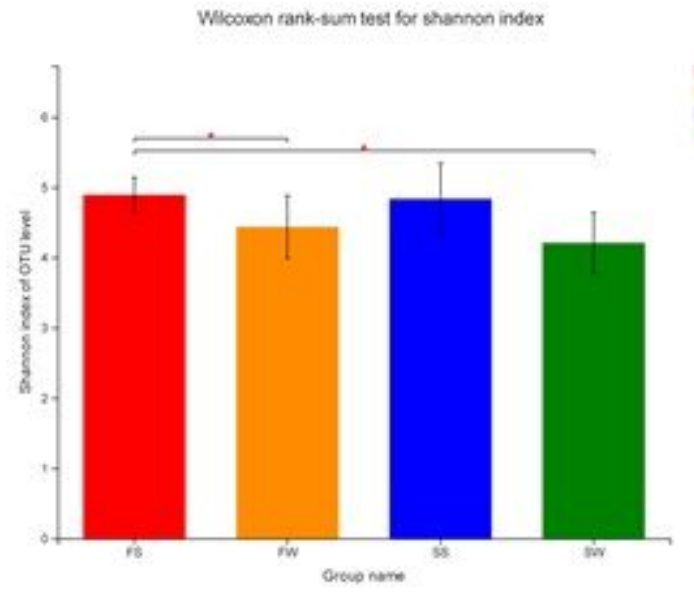

B

Wicowen rank-sum test for chis index

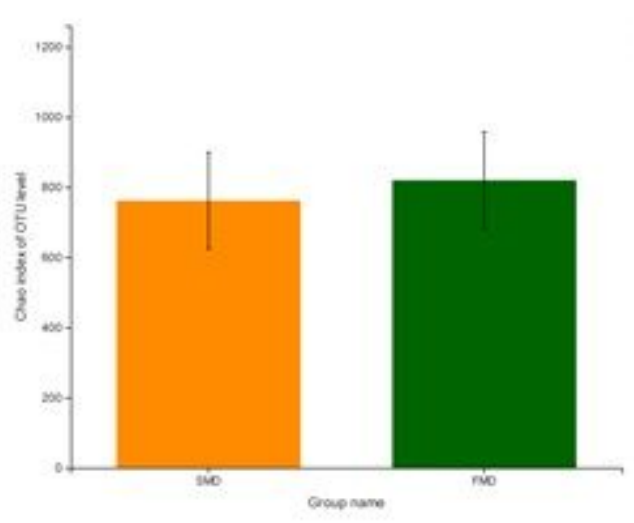

D
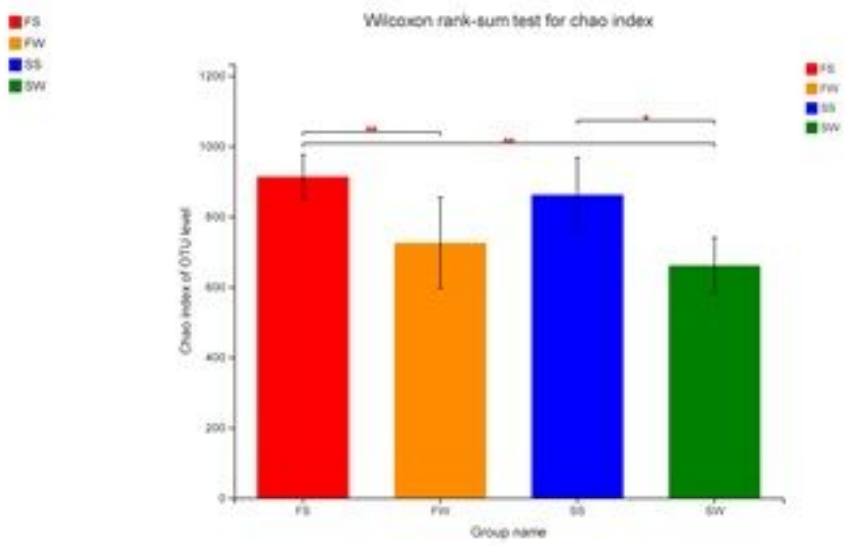

Figure 2

Differences in microbial diversity (Shannon and Chao) between the four groups. Histogram (A) showing the differences in the Shannon index between the SMD and FMD. Histogram (B) showing the differences in the Chao index between the SMD and FMD. Histogram (C) showing the differences in the Shannon index between the four groups. A plot (D) showing the differences in the Chao index between the four groups. Significance was determined using Wilcoxon Rank-sum tests. $(0.01<p \leq 0.05$ marked as *, 0.001 $<\mathrm{p} \leq 0.01$ marked as ${ }^{\star *}, \mathrm{p} \leq 0.001$ marked as $\left.{ }^{\star * \star}\right)$.FW: forest musk deer in the winter; FS: forest musk deer in the summer; SS: Siberian musk deer in the summer; SW: Siberian musk deer in the winter. 


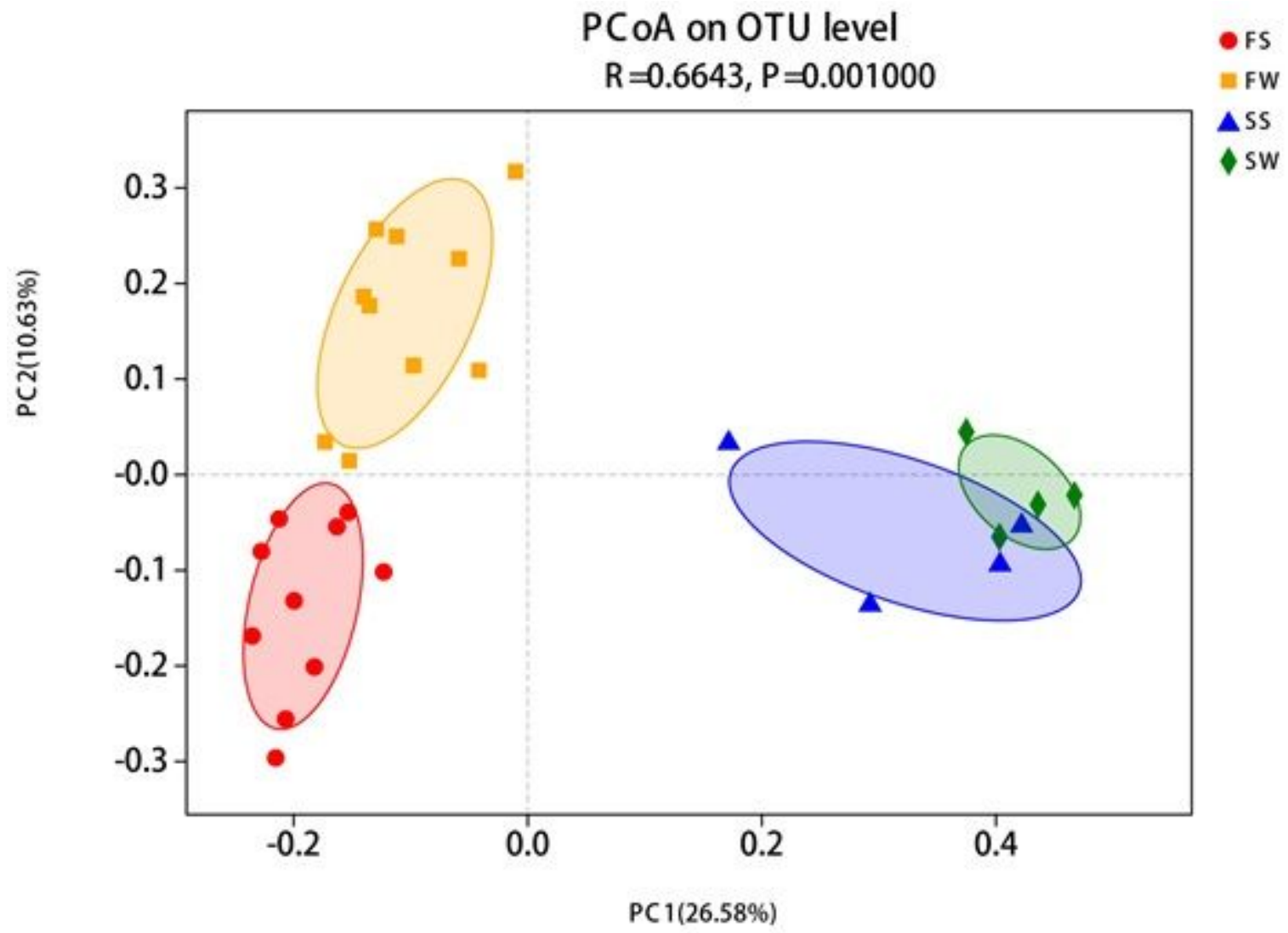

\section{Figure 3}

Non-metric multidimensional scaling ( $\mathrm{PCoA}$ ) plots. To show the distances between the samples based on dissimilarity in the OTU composition of each sample, which was calculated using the Bray-Curtis dissimilarity index. Different colors represent different groups.FW: forest musk deer in the winter; FS: forest musk deer in the summer; SS: Siberian musk deer in the summer; SW: Siberian musk deer in the winter. 
A
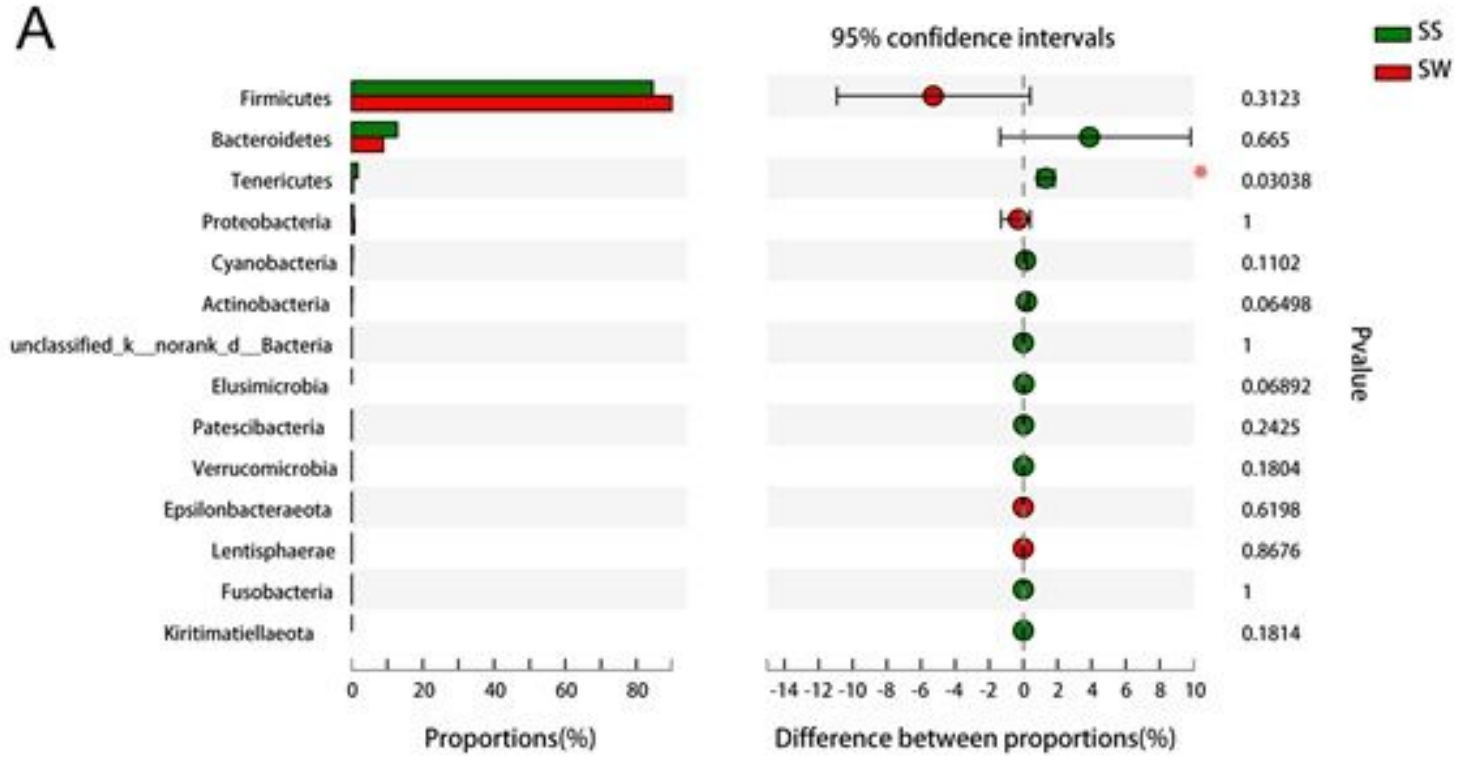

B

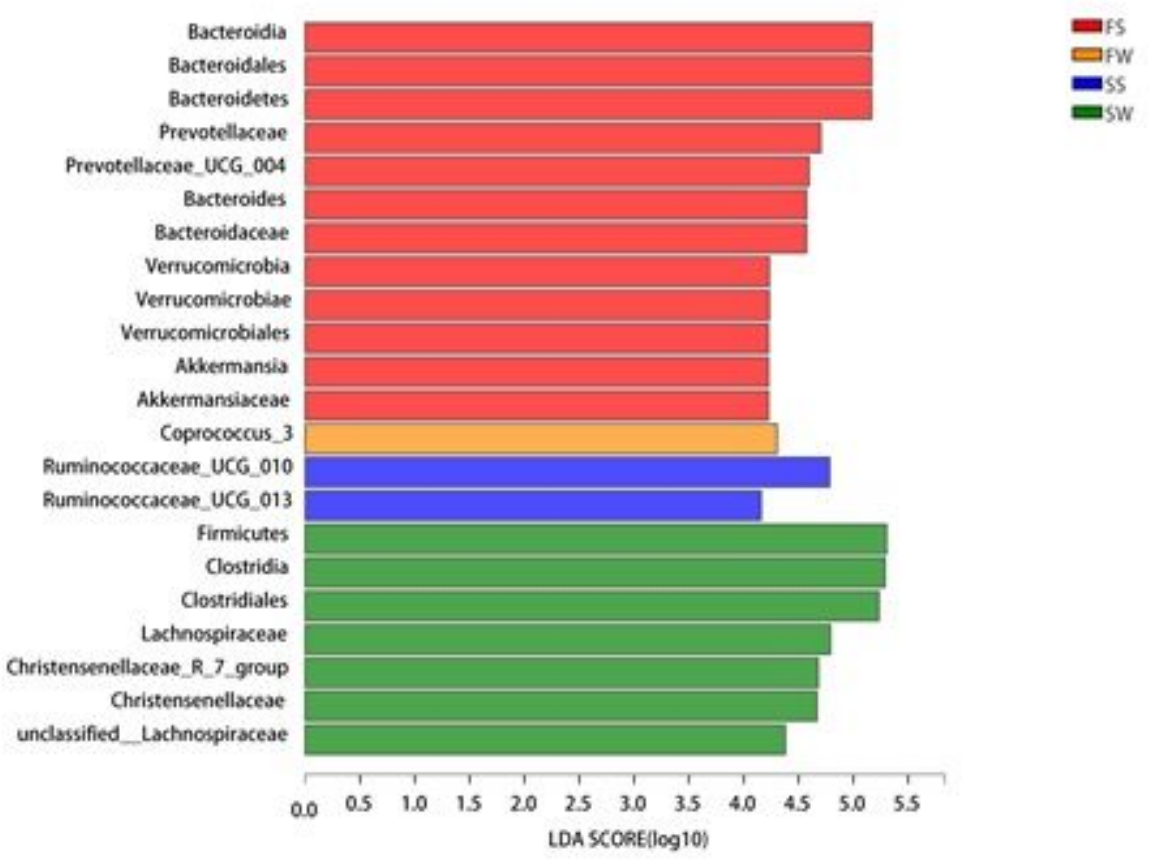

\section{Figure 4}

Seasonal variations in bacterial composition in musk deer. Histogram (A) showing differentially abundant taxa at the phylum level in SMD. Significance was determined using Wilcoxon Rank-sum tests. $\left(0.01<p \leq 0.05\right.$ marked as *, $0.001<p \leq 0.01$ marked as ${ }^{* *}, p \leq 0.001$ marked as $\left.{ }^{\star \star *}\right)$. LDA histogram (B) showing the differences in taxa relative abundance. The length of the bar column represents the LDA score (LDA $>4)$. A larger LDA score indicates a higher contribution of that species to the overall differences in community structure. FW: forest musk deer in the winter; FS: forest musk deer in the summer; SS: Siberian musk deer in the summer; SW: Siberian musk deer in the winter 


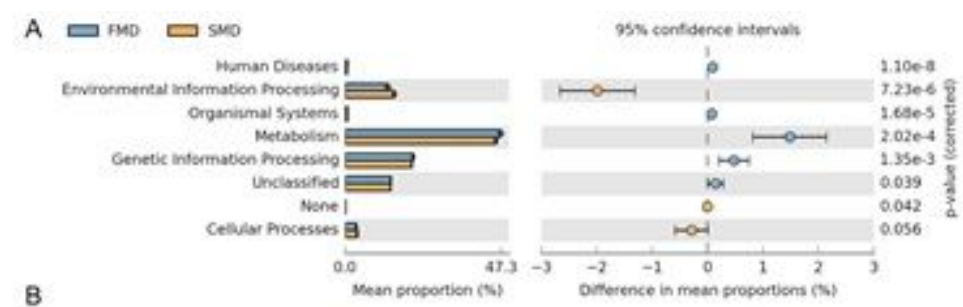

8

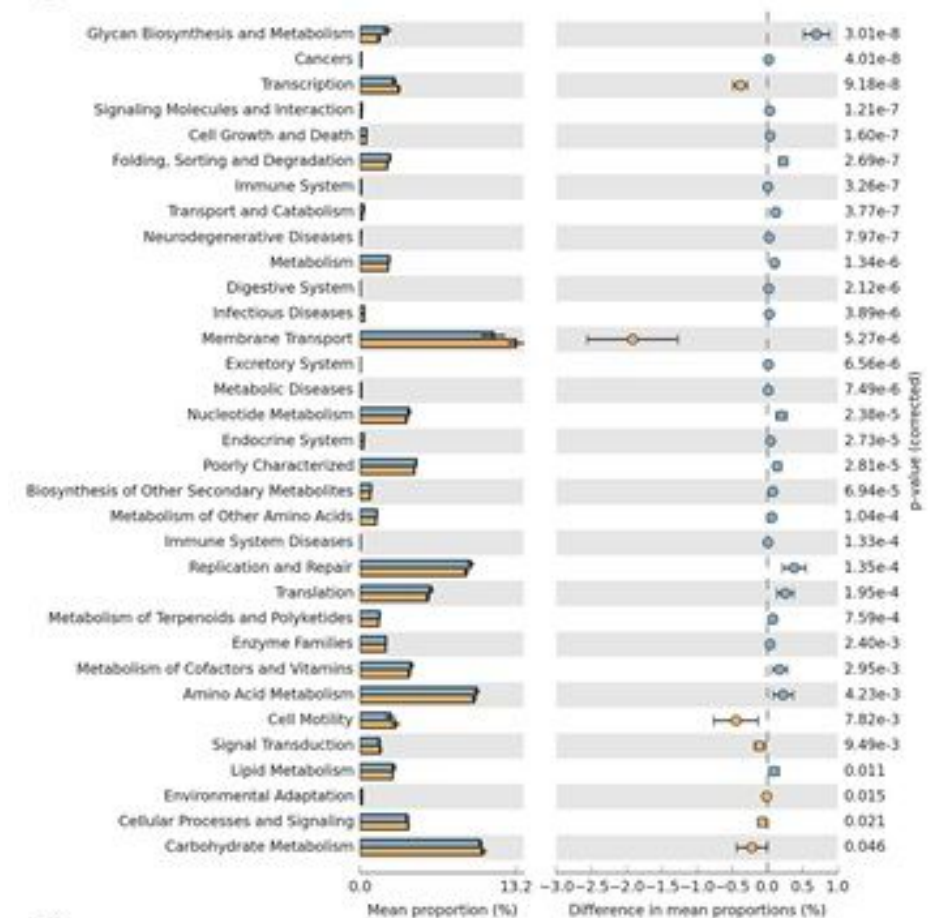

c

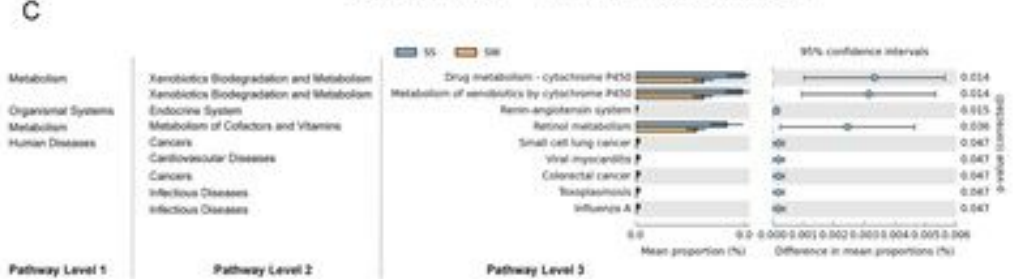

\section{Figure 5}

Predication of microbial function. Histogram (A) comparing functional pathways in SMD and FMD (Level 1). Histogram (B) comparing functional pathways in the FMD and SMD groups (Level 2). Histogram (C) showing seasonal differences in functional pathways in SMD.

\section{Supplementary Files}

This is a list of supplementary files associated with this preprint. Click to download.

- Additionalfile1.docx 